\title{
Financiamento, gasto público e gestão dos recursos em saúde: o cenário de um estado brasileiro
}

\author{
Funding, public spending and management of health resources: \\ the current situation in a Brazilian State
}

\author{
Valéria Rodrigues Leite ${ }^{1}$ \\ Kenio Costa Lima ${ }^{1}$ \\ Cipriano Maia de Vasconcelos ${ }^{1}$
}

${ }^{1}$ Centro de Ciências da

Saúde, Departamento de Odontologia Universidade Federal do Rio grande do Norte. UniversidadeFederal do Rio Grande do Norte. Av. Salgado Filho 1787, Lagoa N ova. 59072-970. Natal RN. leite@ufrnet.br
Abstract Thisarticleinvestigatestheissue of funding and the decentralization process in order to examine the composition, application and management of resources in the healthcare area. The sample surveyed involved 14 municipalities in the state of Rio Grande do N orte, Brazil. The research involved data gathering of financial transfers, the municipality's own resources and primary healthcare expenses. $M$ anagement analysis included a survey of local managers and counselors. It was seen that the U nified Health System is funded mainly by federal transfers and municipal revenues and to a far lesser extent by state resources. Funds have been applied predominantly in primary healthcare. The management process saw centralization of actions in the city governments. M unicipal secretarial offices and councils comply partially with legislation, though they have problems with autonomy and social control. The re sults show that planning and management instruments are limited, due to the contradictions inherent to the institutional, political and cultural context of the region.

Key words Health funding, Decentralization, Public health policies, H ealth management
Resumo 0 artigo aborda a temática do financiamento eo processo de descentralização ten do como objetivo examinar a composição, a direção ea gestão dos recursos na área da saúde. $A$ amostra investigada foi constituída por 14 municípios do Estado do Rio Grande do N orte. A pesquisa envolveu coleta de dados referente às transferências, recursos próprios municipais e gastos com aten ção básica. $\mathrm{Na}$ análise da gestão foi realizado um levantamento junto aos dirigentes locais e consel heiros. O bserva-se que o financiamento do Sistema Ú nico de Saúde se faz às expensas das transferências federais e receitas munici pais, com pequena participação dos recursos estaduais. A direção dos gastos tem privilegiado a atenção básica. N o processo de gestão, verifica-se centralização das ações nas prefeituras. As secretarias municipais e os conseIhos cumprem em parte a legislação e apresentam dificuldades na autonomia e o controle social. Os resultados mostram que os instrumentos de planejamento e gestão são limitados em função das contradições presentes no contexto institucional, político e cultural no âmbito regional.

Palavras-chave Financiamento em saúde, Descentralização, Políticas públicas de saúde, Gestão em saúde 


\section{Introdução}

A temática do financiamento eo processo de descentralização da saúde no Brasil têm povoado a agenda de gestores, movimentos sociais e pesquisadores interessados na consolidação do SistemaÚ nico de Saúde(SUS). Alguns estudos comparativos $\mathrm{s}^{1,2}$ exploraram os desafios da condução nacional da política de saúde em federações que vêm passando por processos de descentralização. Banting e Corbett ${ }^{1}$, ao compararem as implicações do federalismo para as políticas de saúde em cinco países, consideraram como variáveis relevantes a abrangência, o detal hamento ea forma de definição da política no âmbito nacional. Já France ${ }^{2}$ al ega que o desafio da construção de um sistema nacional de saúde em um país federativo é refletir a heterogeneidade das necessidades regionais e, ao mesmo tempo, reduzir as desigualdades para concretizar a cidadania nacional. Por isso, a consideração das peculiaridades do federalismo e da trajetória da política de saúde no Brasil é importante para a discussão do período recente, considerando queo país viveum período deredefinições, voltadas ao delineamento da nova estrutura das relações federativas ${ }^{3}$, com atuação na correção dos desequilíbrios regionais através do processo de descentralização. No que se refere ao Brasil, à classificação que melhor se aplica ao Sistema Ú nico de Saúde (SUS) seria a de descentralização como devolução. Descentralização, nessa perspectiva, implica na ampliação da autonomia das esferas estaduais e municipais, que requisitarão qualificação para a realização de um conjunto de funções públicas4.

Sendo assim, a política de saúde vem desenvolvendo mecanismos próprios que interagem, num contexto específico, com variáveis estruturais e institucionais desenvolvidas pelos atores que atuam nesse setor. No caso, a discussão sobre os aspectos de melhor gestão dos recursos financeiros, merece uma análise mais cuidadosa do processo de descentralização, particularmente da esfera municipal descentralizada da saúde 5 .

Muitos são os trabalhos que buscam analisar o financiamento da saúde no Brasil, quase sempre abordando, de forma agregada, os três níveis da federaçãa ${ }^{6-11}$. Embora se reconheça a enorme heterogeneidade de estados e municípios brasileiros e a particularidade dos gastos com saúde nestas unidades, ainda são poucos os estudos que abordam este tema em contextos regionais ou de estados/municípios específicos, salientando-se aqui os recentes trabalhos de Viana et al. ${ }^{12}$, Fortes et al. ${ }^{13} \mathrm{e}$ M endes et al. ${ }^{14}$.
Assim, 0 artigo pretende contribuir com essa discussão, apresentando o resultado de uma pesquisa sobre a análise do processo de descentralização na área da saúde no Estado do Rio Grande do N orte, respondendo al gumas questões relativas ao setor saúde: Qual a proporção de participação dos entes federativos na composição das receitas da saúde e na direção dos gastos? A alocação dos recursos federais, estaduais e municipais contribui para superar as desigualdades nas disponibilidades de recursos para a saúde? Os recursos de custeio e investimento estão coerentes com as propostas de organização regionalizada e hierarquizada do sistema? Como se define a destinação deinvestimentos? 0 gasto com saúde esta obedecendo ao que determina a resolução do Conselho Nacional de Saúde? A atenção Básica tem sido privilegiada na efetivação do Gasto? Na gestão dos recursos, o dirigente tem autonomia na definição da despesa? O Fundo M unicipal de Saúde funciona conforme previsto na Lei? O Conselho deSaúde participa na definição orçamentária e na fiscalização da utilização dos recursos?

\section{Métodos}

Para responder às questões de pesquisa foram selecionados 14 municípios das 07 regionais de saúde do Estado do Rio Grande do Norte (dois por regional), com base em dois critérios, quais sejam: ser o município com o maior número de habitantes e possuir menos que 5.000 habitantes. Para estes últimos, realizou-se uma escolha aleatória de qual município integraria a amostra, uma vez queem todas as regionais havia mais de um município com o referido critério

Para a elaboração do embasamento teórico, tomou-se como base a questão federativa, e a descentralização, pois em federações, é importante considerar as repercussões da definição de responsabilidades e dos mecanismos de articulação entre esferas de governo para as políticas públicas, além de elementos de natureza institucional (trajetória setorial) ou política (escolhas dos atores em um dado cenário).

Dessa forma, optou-se pelo enfoque das políticas públicas desaúde definidasa partir da constituição de 1988, tomando como base as leis $8.080^{15}$ e $8.142^{16}$, as sucessivas $N$ ormas O peracionais Básicas N O B 01/91, N OB 01/93, N OB 01/96 Norma O peracional deAssistência a SaúdeN OAS 01/01, NOAS 01/02 e a Emenda Constitucional $29^{17}$. 
Para a análise da composição e direcionamento dos recursos, o período do estudo correspondeu aos anos 2003, 2004 e 2005, verificando-se os dados financeiros referenteàs transferências SUS, à despesa com recursos próprios e à atenção básica. Esta análise teve como fonte as informações existentes no SI OPS “um sistema de coleta e processamento de dados sobre receitas totais e gastos com ações e serviços públicos de saúde das três esferas de governo"18. Com relação aos investimentos na área foram coletados dados sobre os convênios federais e estaduais.

Os dados de repasse dos recursos do Governo Federal foram obtidos no M inistério da Saúde, sede estadual, com informações sobre os re cursos do Fundo Nacional de Saúde (FNS) e Fundação $N$ acional de Saúde (Funasa). A coleta dos dados sobre os Convênios Estaduais foi realizada na Secretaria do Estado do Rio Grande do Norte, verificando os convênios celebrados com a Secretaria de Estado da Saúde (Sesap).

Essas informações foram agregadas em uma única fonte, um banco de dados, apresentando informações referentes às regiões e à população, os diversos componentes individualizados eagregados (somatórios) das Despesas com Saúde na Subfunção Administrativa Direta, Subfunção Vinculadas e I nformações Complementares.

Embora tenham sido coletados todos os dados sobre os recursos direcionados para as funções supracitadas, na análise final, para uma melhor compreensão, optou-se trabalhar apenas as variáveis que respondem às questões de pesquisa. Desta forma a análise está fundamentada na despesa total com município, despesa com recursos próprios, transferências SUS, atenção básica, convênio federal e convênio estadual.

Para a identificação da gestão e a equidade do sistema, o processo de investigação, ocorrida em 2006 e 2007, partiu do levantamento das informações relativas aos municípios selecionados, através de pesquisa eletrônica no caderno de informação da secretaria executiva do M inistério da Saúde, elaborando um diagnóstico sobre os municípios selecionados. Posteriormente, efetuou-se uma consulta na Secretaria M unicipal da Saúde, aos documentos ${ }^{19}$ relativos ao Plano Municipal de Saúde, Plano Plurianual (PPA), Lei deDiretrizes Orçamentária (LDO), Lei Orçamentária Anual (LOA), Programação Pactuada Integrada (PPI) eo Relatório de Gestão (resultados).

Paralelamente, foi analisado o Fundo M unicipal de Saúde tomando como base a Portaria de $\mathrm{n} 0$ 548, de 12 deabril de2001, ondeestabeleceque o orçamento deva respeitar as políticas e os pro- gramas de trabalho das Secretarias de Saúde, observando a Agenda de Saúde o o Plano de Saúde, elaborados e aprovados pelos respectivos Conselhos de Saúde.

Posteriormente após a análise desses instrumentos de planejamento e gestão foi elaborado um levantamento das $\mathrm{Atas}^{20}$ do Conselho Municipal de Saúde verificando sua atuação na elaboração e participação nos instrumentos de planejamento. Em seguida foram realizadas as entrevistas semiestruturadas, individual e em grupo com pessoas selecionadas. A seleção foi de acordo com os critérios de ocupação de cargo, onde foram entrevistados quatorze secretários municipais e representantes do Fundo Municipal de Saúdee quarenta e dois conselheiros municipais.

\section{Resultados}

Com relação à análise da composição da despesa total, os dados apresentaram equivalência dos recursos provenientes do governo federal (Figura 1), repasse das transferências SUS, e do governo municipal, através dos recursos próprios ( $\mathrm{Fi}$ gura 2), sem que tenha havido diferença significativa para o conjunto dos municípios estudados. Esse resultado indica uma tendência à uniformidade eao declínio na variabilidade, seconsiderados os anos de 2003 e2004. Na direção dos gastos, o recurso que apresentou maior representatividade na amostra foi a Atenção Básica (Figura 3) e não ocorre homogeneidade na sua aplicação. Os recursos provenientes de convênios estaduais apresentaram uma mediana baixa

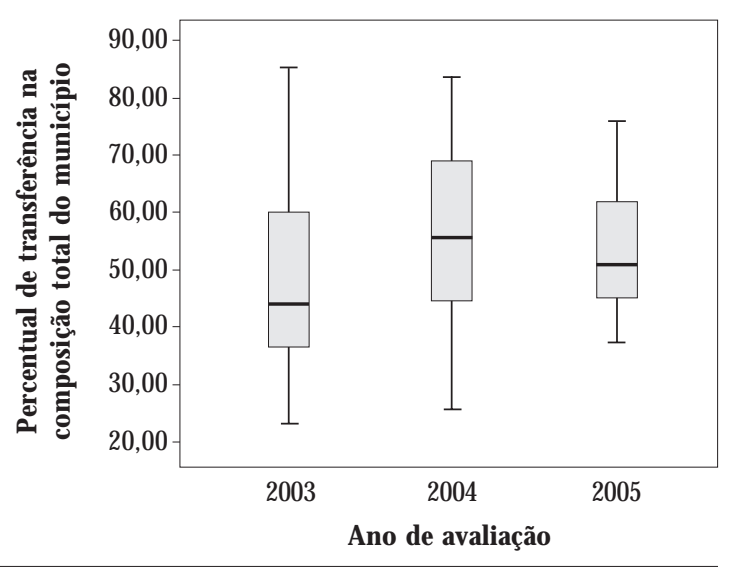

Figura 1. Recursos de Transferência na composição da despesa total. 
(Tabela 1) e retrata a inexistência da aplicação de recursos em investimento ea pouca participação do estado na composição das receitas da saúde. Tal comportamento indicou homogeneidade durante o período estudado.

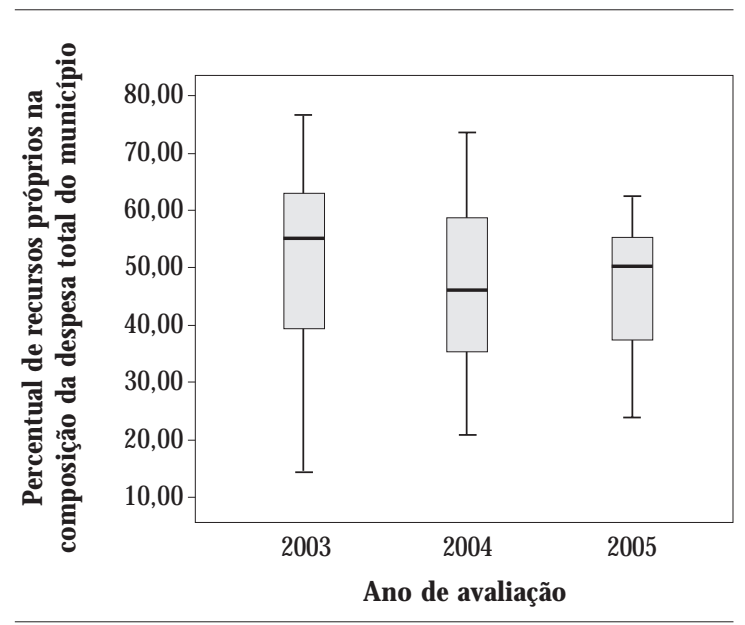

Figura 2. Recursos próprios na composição da despesa total

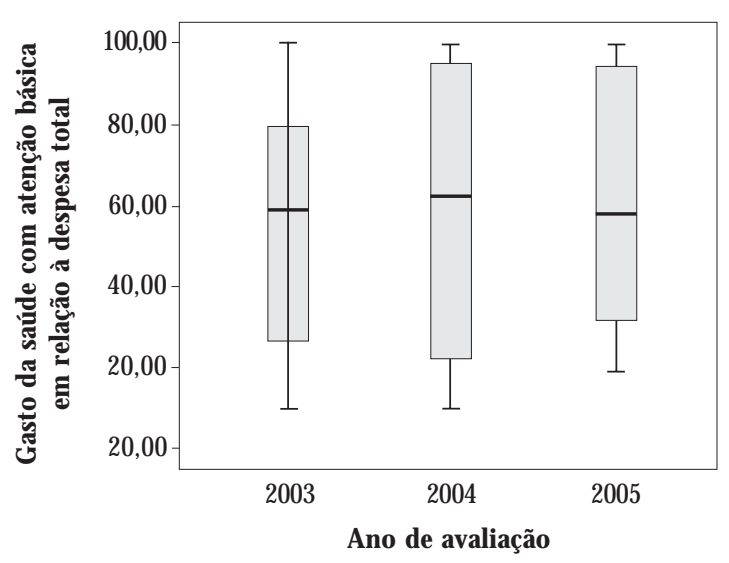

Figura 3. Gasto com atenção básica em relação à despesa total.

Tabela 1. Recursos provenientes do Convênio Estadual com relação à Despesa Total.

\begin{tabular}{rrrccc}
\hline Ano & Mediana & Q25-Q75 & $\begin{array}{c}\text { Soma dos } \\
\text { postos }\end{array}$ & Fr & $P$ \\
\hline 2003 & 0 & $0-3,68$ & 25,5 & 3,389 & 0,1837 \\
2004 & 0 & $0-1,81$ & 26,0 & & \\
2005 & 0,26 & $0-3,15$ & 32,5 & & \\
\hline
\end{tabular}

Fonte: Secretaria do Estado do Rio Grande do N orte
No que se referem à gestão, as secretarias municipais de saúde, apesar de estarem amparadas pelos instrumentos de planejamento, na maioria das vezes, apresentam-se como simples prestadoras de serviços. Os instrumentos de planejamento são insuficientes, uma vez que, quando existem, apontam basicamente os objetivos e metas, focado nas exigências normativas, e não retrata a realidade local.

$\mathrm{N}$ a autonomia setorial, pode-se dizer queexiste uma concentração das ações de saúde nas secretarias de finanças das prefeituras, principalmente na questão orçamentária, decorrente da ausência de estrutura nas secretarias municipais. Na maioria dos municípios, o planejamento dos recursos é elaborado pelas prefeituras, especificamente pelo contador, e em alguns casos, a direção dos recursos não indica a necessidade da população.

Essa constatação pode ser feita partindo da análise documental, elaborada na pesquisa de campo, onde se verifica que na maioria dos municípios, a Proposta Orçamentária apresenta-se em conformidade com o Plano de Saúde, porém não traduz as necessidades da população, uma vez que não são realizadas pesquisas e estudos de demanda, e a articulação com os poucos técnicos na área da saúde e com o Conselho Municipal de Saúde é praticamente inexistente. Muitas vezes esses documentos são basicamente repetições das ações dos anos anteriores, comprometendo 0 Art. 36 da Lei 8.080/90, que estabelece 0 plano de saúde e a proposta orçamentária.

Os demonstrativos orçamentários financeiros constituem materiais essenciais para 0 acompanhamento, e a fiscalização por parte do conseIho (lei 8.080-Art. 33), porém este enfrenta problemas: as informações demoram a ser repassada e al gumas vezes nem ocorre. Os consel heiros não têm capacidade para interpretar os dados, não existe compromisso com relação às suas responsabilidades, ocorrendo substituições constantes na equipe, o que dificulta treinamentos eal gumas vezes existem divergências políticas entreconselheiros e prefeito. Sendo assim, o processo de planejamento eorçamento (art. 36) não éfundamentado em sugestões dos órgãos deliberativos.

A atuação dos Consel hos M unicipais, em todos os municípios, apesar do suporte legal, não cumpre o seu papel, na medida em que apresenta diversas dificuldades. Não dispõe de pessoal qualificado para as exigências da lei, principalmente no que diz respeito ao cumprimento da fiscalização orçamentária. Os cursos de capacitação não são suficientes para uma atuação que 
responda o cumprimento da lei. Outro ponto a ser considerado é a falta de compromisso dos conselheiros e a renovação do mandato, dificultando a continuidade dos participantes que já tem algum entendimento.

A fiscalização por partedo conselho (lei 8.080Art. 33) enfrenta problemas. Existe, em parte, o cumprimento das secretarias (prefeituras) no que diz respeito aos instrumentos de planejamento, porém os consel hos municipais apresentam-se à margem desse processo. A articulação com a secretaria, prefeitura eaté mesmo com a população éinsuficiente ou inadequada, particularmenteno que se refere ao planejamento e orçamento. Sendo assim, esses instrumentos não são fundamentados em sugestões dos órgãos deliberativos, 0 que provavelmente não traduz a realidade local.

Uma análise abrangente do processo com base na combinação dos vários olhares permite identificar queem geral os princípios e as diretrizes do SUS são seguidos nas ações municipais e apresentam universalidade de acesso aos serviços. Porém, ao assumir a gestão de recursos e as responsabilidades inerentes à gestão da saúde local, a maioria dos municípios não estava com capacidade institucional para assumir as tarefas exigidas pelas diretrizes da descentral ização, comprometendo a implementação das atividades com relação à integralidade e equidade.

\section{Discussão}

Em decorrência da descentralização, na política de saúderegistram-se, no Brasil, nosúltimos vinte anos mudanças nos cinco atributos relevantes para a caracterização do federalismo destacados por 0 binger et al..$^{21}$ : "os arranjos institucionais e regras para decisões nacionais com vistas a incorporar interesses territoriais (por meio das comissõesintergovernamentaisna saúde); o conjunto de atores com base territorial (ampliação dos atores envolvidos na implementação da política, como gestores locais e conselheiros de saúde); os arranjos legais para definir responsabilidades entreníveis degoverno (leis da saúde, normas operacionais do SUS e outras portarias); os arranjos de transferências fiscais intergovernamentais (mecanismos de financiamento, tipos e volume das transferências federais, mudanças na participação das esferas de governo no gasto em saúde); os arranjos informais entre governos, verticais e horizontais ( relações entregestores do SUS egovernantes)". A pesar do esforço para atender esses atributos e consequentemente as exi- gências da descentralização al gumas observações, a partir desse estudo, podem ser levantadas.

As transferências federais voltadas para a equalização, ora das receitas disponíveis, ora das necessi dades de gastos, ainda apresentam-se relevantes na composição dos recursos disponíveis para a saúde. Isto representa, consequentemente, uma maior responsabilidade da esfera federal na definição das regras e diretrizes da aplicação dos recursos, indicando que o atual modelo de descentral ização seencontra em fasedeconformação e acomodação.

A relevância dos recursos federais ${ }^{22}$ não favorece uma maior equidade no gasto público em saúde por que: 1) não permitem, na sua mai oria, a redistribuição de recursos para estados e municípios com maiores dificuldades orçamentários, pois sua forte associação com a oferta e a produção impedeampliar as receitas disponíveis em estados e municípios com menor capacidade de arrecadação. 2) desconsideram as possibilidades reais de aporte de recursos e ampliação de gasto a partir das receitas próprias das esferas subnacionais, fruto das regras que informam 0 federalismo fiscal no Brasil.

A pesar da relevância dos recursos federais, 0 avanço da implementação das políticas de saúde resultou na queda relativa dos recursos da União ${ }^{23}$ e maior partici pação dosmunicípios no financiamento. Ainda que tenha havido uma diminuição da participação do governo federal no financiamento da saúde pública, ela não foi acompanhada da redução de sua presença na determinação da política, principalmente em nível de atenção básica.

Estudo realizado por Campelli e Calvo ${ }^{10}$ no período de 2000 a 2003 onde foram avaliadas as Unidades Federativas do Brasil mostra que após a EC - 29 os gastos públicos apresentam uma tendência crescente nas aplicações dos recursos em saúde. "A despesa por habitante, que era de $\mathrm{R} \$$ 201,20 em 2000, aumentou para $\mathrm{R} \$ 304,18 \mathrm{em}$ 2003. Porém, a U nião vem reduzindo sua participação relativa no financiamento da saúde após a EC-29. Em 2000 sua participação era de 59,8\%; em 2001 de 56,2\%, em 2002 de 53,1\% eem 2003 de $48 \%$ ". Constata-se que, se de um lado há uma diminuição da partici pação da União, por outro, há o consequente aumento relativo dos gastos em saúde para os Estados e M unicípios.

O Rio Grande do Norte apresenta cenário semelhante. $\mathrm{Na}$ composição da Despesa Total com saúde, os recursos provenientes do governo federal (transferências) e do governo municipal (recursos próprios) apresentam equivalência. A 
participação dos recursos federais, na composição dos recursos públicos de saúde, apesar de significativa, vem reduzindo gradativamente. Em 2003, essepercentual ficou abaixo de $50 \%$, invertendo a composição do financiamento.

Os resultados apontam para o aumento da participação dos municípios na composição dos recursos, enquanto que a participação do estado éinsignificante. M ais responsabilidades e aportes financeiros ficaram sob a égide dos municípios.

Porém, as transferências de recursos e de responsabilidades, do governo federal para o municipal, não foi acompanhado de investimentos mais substantivos na rede pública de serviços. Os escassos recursos são destinados na maioria das vezes para a manuten ção dos equipamentos. Observa-se que existe precariedade na aplicação de inversões e no capital fixo, o que dificulta a implantação do SUS. O direcionamento não esta coerente com as propostas deorganização regionalizada ehierarquizada. Além de ser muito baixo, não existeplanejamento para a aplicação baseado nas necessidades locais. São necessários novos investimentos para permitir uma oferta de saúde adequada.

Com relação à direção dos recursos em termos de finalidades, estes obedecem ao que determina a resolução do Conselho $N$ acional de Saúde (CNS). A Atenção Básica vem sendo privilegiada na direção dos recursos, porém não ocorre uma homogeneidade na aplicação dos mesmos. Embora esse movimento seja importante e na direção correta, somenteindica quehouve ampliação dos serviços, mas não o suficiente, uma vez quea aplicação dos recursos nos municípiosse diferencia enão são suficientes para cobrir a demanda e, consequentemente, os princípios da equidade. De acordo com $\mathrm{M}$ arques $^{24}$, a ampliação do campo de ação da Atenção Básica indica o crescimento da importância da estratégia da política de saúde implementada pelo M inistério da Saúde.

$\mathrm{Na}$ apreciação do processo de planejamento e gestão, no estado, assim como identificado em trabalho realizado por $\mathrm{M} \mathrm{endes}^{5}$, verifica-se que houve variações nas estratégias de descentralização, associadas a movimentos de reconfiguração das funções dos municípios na implantação das políticas de saúde.

No entanto, a descentralização per se não é suficiente para a concretização do SUS. Os seus avanços efetivos estão intimamenterelacionados a outros aspectos relevantes para a consolidação do sistema, como o adequado aporte de recursos financeiros, o fortalecimento da capacidade gestora nos três níveis de governo e a permeabi- lidade das instituições do setor saúde aos valores democráticos ${ }^{25}$.

A descentralização $0^{12}$ ao permitir a ampliação significativa detransferências de recursos emaior poder de arrecadação a estados emunicípios, não estabeleceu um processo pactuado de redefinição defunções ecompetências de gestão na área social. Constata-sea necessidade de adotar iniciativas com vistas a aumentar o aprimoramento desses mecanismos, tornando mais efetivas a participação social e o controle público sobre a utilização dos mesmos e aumentar a transparência, a eficácia e a equidade na utilização dos recursos.

Embora ocorram iniciativas de acompanhamento e de avaliação através do M inistério da Saúde e da Organização Pan-Americana em indicar diretrizes para formuladores de política e gestores desses instrumentos ainda permanece uma lacuna entre o que é planejado e o que é implementado.

A aproximação do SUS real ao SUS inscrito na legislação, entretanto, além demedidas incrementais, necessita de iniciativas que possam corrigir distorções e contradições, a exemplo dos mecanismos de repasse de recursos, e qualificar os processos de gestão. Isso exige, necessariamente, um novo pacto, capaz de ampliar o grau de responsabilidades entre gestores, sobretudo nos espaços local eestadual, quejuntos, com o apoio do M inistério da Saúde e de programas de Cooperação Técnica - CT devem construir as múltiplas conexões no âmbito regional ${ }^{4}$.

É importante identificar as ações do M inisté rio da Saúde como estratégia instrumental para fomentar a capacidade dos estados e municípios do país, promovendo o desenvolvimento nacional autônomo da saúde, com referência aos princípios deigualdade, eficácia, eficiência, sustentabilidade e participação. Adotar programas que fortaleçam a representação evisem o alinhamento dos recursos humanos, financeiros e tecnológicos, em busca de estabelecer processos e mecanismos para que a CT aconteça de forma planejada, organizada e participativa.

Esses programas podem estar relacionadosaos conceitos de desenvolvimento institucional e organizacional, compreendendo os processos demeIhoramento global à procura de um melhor desempenho deseusresultados. N essesentido, sefaz necessário considerar a definição das políticas internas e externas, da estrutura e do funcionamento organizacional e do estilo de gestão; de acordo com os fatores externos que condicionam o trabalho da organização; eoutros aspectos-chavepara estabelecer o funcionamento da instituição. 
Desenvolver, a nível local, ações de planejamento e permitir melhor monitoramento, controle, avaliação e informe dos resultados, a fim de contribuir para um resultado esperado regional. N essesentido, énecessário ampliar a presença e a capacidade das instituições para apoiar programas e prioridades nacionais, mediante 0 fortalecimento dos níveis estaduais/municipais. M anter uma estreita relação política, estratégica e técnica com parceiros mediante a definição de redes de relacionamento de instituições públicas eprivadas, permitindo que cada integrante mantenha sua independência em relação à rede e aos demais integrantes, para compartilhar objetivos e decisões e permitir que cada ator possa contribuir para a execução da cooperação.

Faz-se necessário "apostar na capacidade de organização dos Conselheiros de Saúde das três esferas de gestão do SUS", investir na capacitação de seus agentes, criando redes de informações e de troca de experiências. Tais iniciativas são de terminantes para que "os recursos mínimos para a saúde previstos na EC-29 sejam realmente assegurados nas leis orçamentárias, para que sejam empenhados em tempo hábil, sem contingenciamentos e sejam executados". Os Conselhos de Saúde têm agora referência de valores financeiros para aprovarem ou não as prestações de contas do SU S².
É importante fazer alusão ao papel a ser desempenhado pelo controle social na efetivação deste dispositivo constitucional, queé, apesar de todas as dificuldades, o melhor caminho para a consolidação do SUS. As considerações de Rezende ${ }^{26}$ também apontam para essa direção, ao destacar queé necessário "apostar na capacidade de organização dos Conselheiros de Saúde das três esferas de gestão do SUS". A vigilância do Tribunal deContas e do M inistério Público pode configurar um Controle Social mais efetivo ${ }^{10}$.

Além disso, a adesão ao Pacto pela Saúde, em fevereiro de 2006, coloca para a gestão do SUS nos municípios um conjunto de responsabilidades e compromissos sanitários, com o objetivo de fortalecer a capacidade gestora do sistema de saúde. Essa situação está expressa tanto na pactuação das prioridades, objetivos emetas do Pacto pela Vida, quanto na discussão das responsabilidades do Pacto de Gestão.

0 debate, mais uma vez, está aberto. As divergências, entretanto, não devem servir para distanciar, mas para produzir o consenso entre todosaquelesquepropugnam pelo fortalecimento do SUS. A publicação dos resultados da investigação deverá contribuir com a discussão sobre os mecanismos de alocação de recursos e a formulação da política de saúde, planejamento e gestão, no âmbito estadual emunicipal, embasadas nas análises realizadas.

\section{Colaboradores}

VR Leite, KC Lima e CM Vasconcelos participaram igualmente detodas as etapas de elaboração do artigo. 


\section{Referências}

1. Banting K, Corbett S. Federalism and health care policy: an introduction. In: Health Policy and Federalism Workshop; 2001 Oct15-16; São Paulo, Brazil.

2. France $G$. Compatibilità fra il federalismo e gli standard sanitari nazionali. In: France G, organizador. Federalismo, regionalismo e Standard Sanitari NazionaIi. Milano: Dott. A Gluffré Editora; 2001. p. 169-197.

3. Nóbrega M. Federalismo seletivo e equalização fiscal: o novo modelo de gestão fiscal e o impacto nos entes subnacionais. In: M endes GF, organizador. São Paulo: Saraiva; 2005.

4. Pasche D F, Righi L B,Thome HI, Stolz ED. Paradoxos das políticas de descentralização de saúde no Brasil. Rev Panam Salud Publica 2006; 20(6):416422.

5. M endes NA. Financiamento, gasto e gestão (SUS). A gestão descentralizada semiplena e plena do sistema municipal no Estado de São Paulo (1995-2001) [tese]. 2005. Campinas: UNICAM P; 2005.

6. M édici A. Gastos com saúde nas três esferas de governo: 1980-1990: o financiamento da saúde no Brasil. Brasília: Organização Pan-Americana da Saúde; 1994. (Série Economia e Financiamento, 4)

7. Costa RCR. Descentralização, financiamento e regulação: a reforma do sistema público de saúde no Brasil durante a década de 1990. Rev Sociol Polít 2002; (18):49-71.

8. Faveret ACSC. A vinculação constitucional de recursos para a saúde: avanços, entraves e perspectivas. Cien Saude Colet 2003; 8(2):371-378.

9. U gá MAD, Piola SF, Porto SM, Vianna, SM. Descentralização e alocação de recursos no âmbito do Sistema Ú nico de Saúde (SUS). Cien Saude Colet 2003; 8(2):417-437.

10. Campelli MGR, Calvo M CM . O cumprimento da Emenda Constitucional $n^{\circ} .29$ no Brasil. Cad Saude Publica 2007; 23(7):1613-1623.

11. Dain S. Os vários mundos do financiamento da saúde no Brasil: uma tentativa de integração. Cien Saude Colet 2007;12(Supl.):1851-1864.

12. Viana ALd'A, Novaes HMD, Silva JA, Rocha JSY, Ibañez N, Elias PEM. Financiamento e gasto com saúde nos municípios paulistas com população superior a 100 mil habitantes (2000-2003). São Paulo: Centro de Estudos de Cultura Contemporânea Consórcio M edicina USP; 2006. (Cadernos de Atenção Básica: Estudos Descritivos, 2).

13. Fortes FBCP, Machado ENM, Matias BD. Gasto público com saúde nos municípios de $\mathrm{M}$ inas $\mathrm{Ge}$ rais: 2000-2002. Divulg Saúde D ebate 2007; 37:69-89.

14. Mendes A, Kayano J, Heimann LS, Junqueira V Castro IEN, Ferreira MRJ, RM Sena. Apuração do gasto SUS regionalizado no Estado de São Paulo. São Paulo: Instituto de Saúde; 2008 (Temas em Saúde Coletiva, 6).

15. Brasil. Lei Orgânica da Saúde - Lei no 8080, de 19 de setembro de 1990. Dispõe sobre as condições para a promoção, proteção e recuperação da saúde, a organização e o funcionamento dos serviços correspondentes e dá outras providências. [acessado 2006 maio 1]. Disponível em: http://www.senado. gov.br/sicon/ExecutaPesquisaLegislacao.action.
16. Brasil. Lei no 8142, de 28 de dezembro de 1990. Dispõe sobre a participação da comunidade na gestão do Sistema Ú nico de Saúde - SUS e sobre as transferências intergovernamentais de recursos financeiros na área da saúde e dá outras providências. [acessado 2006 maio 1]. Disponível em: http://www.senado. gov.br/sicon/ExecutaPesquisaLegislacao.action.

17. Brasil. Emenda Constitucional $n .029$, de 13 de setembro de 2000. Altera os artigos 34, 35, 156, 160, 167 e 198 da Constituição Federal e acrescenta artigo ao Ato das Disposições Constitucionais Transitórias, para assegurar os recursos mínimos para o financiamento das ações e serviços públicos de saúde. [acessado 2006 maio 01]. Disponível em: http://www. presidencia.gov.br/ccivil/Constituicao/Emendas/ Emc/emc29.htm]

18. Brasil. Sistema de Informações sobre Orçamentos Públicos em Saúde. Afinal, o que é o SIOPS? [acessado 2006 mar 25]. Disponível em: http://www.siops. datasus.gov.br.

19. Rio Grande do Norte. Prefeituras Municipais. Documento Oficiais das Prefeituras Municipais de Saúde. Plano Plurianual, Plano Anual, Relatório de Gestão 2003 a 2005.

20. Rio Grande do Norte. Documentos Oficiais do Conselho Municipal de Saúde - Atas das reuniões, Plano Anual, Relatório de Gestão 2003 a 2005.

21. Obinger $H$, Leibfried $S$, Castles F. Federalism and the Welfare State. Cambridge: Cambridge University Press; 2005.

22. Lima LD. Conexões entre o federalismo fiscal e o financiamento da política de saúde no Brasil. Cien Saude Colet 2007; 12(2):511-522.

23. Bueno WS, M erhy EE. Os equívocos da NOB/96: uma proposta em sintonia com os projetos neoliberalizantes? In: 10 ${ }^{a}$ Conferência Nacional de Saúde. Tema: Norma Operacional Básica 01/96, 1997. (M imeo).

24. Marques RM, M endes $A$. A política de incentivos do M inistério da Saúde para a atenção básica: uma ameaça à autonomia dos gestores municipais e ao princípio da integralidade? Cad Saude Publica 2002; 18(Supl.):163-171.

25. Levcovitz E, Yamamoto EK, Silva LAD. Notas sobre a crise de financiamento do setor público no âmbito do SUDS. Saúde Debate 1989; (27):12-17.

26. Rezende C. N ota técnica: a implementação da Emenda Constitucional 29 (Emenda da Saúde). [acessado 2007 mar 18]. Disponível em: http://www.pt. org.br

Artigo apresentado em 05/04/2011

Aprovado em 16/04/2011

Versão final apresentada em 06/08/2011 